\title{
Effect on the Tritium Breeding Ratio for a distributed ICRF antenna in a DEMO reactor
}

\author{
A. Garcia ${ }^{1,2,3,4 \text { a) } J-M ~ N o t e r d a e m e}{ }^{1,4}$ U. Fischer $^{2}$ J. Dies $^{3}$ \\ ${ }^{1}$ Max-Planck-Institut für Plasmaphysik (IPP), Garching, Germany \\ ${ }^{2}$ Karlsruhe Institute of Technology (KIT), Karlsruhe, Germany \\ ${ }^{3}$ Polytechnic University of Catalonia (UPC), Barcelona, Spain \\ ${ }^{4}$ Department of Applied Physics, Ghent University, Ghent, Belgium \\ a)albert.garcia.hp@gmail.com
}

\begin{abstract}
This thesis reports results of MCNP-5 calculations, with the nuclear data library FENDL-2.1, to assess the effect on the Tritium Breeding Ratio (TBR) due to a distributed Ion Cyclotron Range of Frequencies (ICRF) antenna integrated in the blanket of a DEMO fusion power reactor. A representative configuration of both a conceptual antenna design and the DEMO reactor was used together with a parametric analysis for different parameters that strongly affect the TBR. These are the type of breeding blanket (Helium Cooled Pebble Bed, Helium Cooled Lithium Lead and Water Cooled Lithium Lead), the covering ratio of the straps of the antenna (the ratio between the surface of all the straps and the projected surface of the antenna slot: $0.49,0.72$ and 0.94$)$, the antenna radial thickness $(20 \mathrm{~cm}$ and $40 \mathrm{~cm})$, the thickness of the straps $(2 \mathrm{~cm}, 4 \mathrm{~cm}$ and a double layer of $0.2 \mathrm{~cm}$ plus $2.5 \mathrm{~cm}$ with the composition of the First Wall), and finally the poloidal position of the antenna $\left(0^{\circ}\right.$, which is the equatorial port, $40^{\circ}$ and $90^{\circ}$, which is the upper port). For an antenna with a full toroidal circumference of $360^{\circ}$, located poloidaly at $40^{\circ}$ with a poloidal extension of $1 \mathrm{~m}$ and a total First Wall surface of $67 \mathrm{~m}^{2}$, the reduction of the TBR is $-0.35 \%$ for a HCPB blanket concept, $-0.53 \%$ for a HCLL blanket concept and $-0.51 \%$ for a WCLL blanket concept. In all cases covered by the parametric analysis, the loss of TBR remains below $0.61 \%$. Such a distributed ICRF antenna has thus only a marginal effect on the TBR for a DEMO reactor.
\end{abstract}

\section{INTRODUCTION}

A DEMO fusion power reactor [1] must be self-sufficient in tritium. Hence, a net Tritium Breeding Ratio (TBR - the ratio of $\mathrm{T}$ produced in the blanket to the $\mathrm{T}$ consumed in the core reactions) greater than one is essential. Some margin is required to account for tritium losses and uncertainties. The typical design targets the global TBR required in the range of 1.05 to 1.15 [2]. The integration of diagnostics, plasma heating components, etc. deteriorates the TBR due to the required replacement of breeder blanket parts by non-breeding materials or volumes. Recently, a new concept for the Ion Cyclotron Range of Frequencies (ICRF) antenna integrated within the blanket has been proposed [3] (see Figure 1). From the ICRF engineering and physics point of view, the in-blanket IC array presents large advantages (low power density, no use of equatorial ports, better definition of the k-spectrum), but the impact on the TBR is less evident. The antenna uses a large surface but, in contrast to a port opening, it does not require a large volume at expense of the blanket breeder. The effect on the TBR is due to the combination of three effects:

1. Parasitic absorption of neutrons in the antenna

2. Moderation of neutrons affecting the neutron spectrum

3. A reduction of the total breeding blanket volume 
The objective of this paper is to quantify this loss of TBR and thus to check if such an antenna is compatible with the TBR requirements of DEMO. The assessment has been performed for the European DEMO power reactor and three types of breeder blanket concepts, the Helium Cooled Pebble Bed (HCPB) [4], the Helium Cooled Lithium Lead (HCLL) [4] and the Water Cooled Lithium Lead (WCLL) [5]. The calculations were done at KIT (Karlsruhe Institute of Technology) using the Monte Carlo N-Particle code MCNP, version 5 [6], and nuclear cross-section data from the Fusion Evaluated Nuclear Data Library (FENDL-2.1) [7].

The antenna used is a preliminary conceptual version and it is still being developed. This study is a complement to this development in order to ensure its feasibility in terms of tritium self-sufficiency. In case the preliminary version needs to be biased, a parametric analysis consisting in some variations of the first design has been performed. The parameters considered in this parametric analysis were chosen following the criteria of being likely to be biased, on one hand, and directly affect the TBR, on the other hand. These are, in addition to the breeding blanket concepts, the covering ratio of the straps of the antenna, the radial thickness of the antenna, the thickness of the straps and the poloidal position of the antenna. All them have been varied and the loss of TBR has been estimated.

Other physics or engineering design aspects are not considered within this work.

\section{BLANKET}

The European fusion technology programme considers two blanket development lines which will be tested in ITER [8], the HCPB blanket with Lithium ceramics pebbles as breeder material and beryllium pebbles as neutron multiplier, and the HCLL blanket with the Pb-Li eutectic alloy acting both as breeder and neutron multiplier. Both, as well as the WCLL blanket, a very strong candidate which is especially attractive for using water as coolant, are considered in this paper. For the Monte-Carlo simulations described in Section 4 the reference model will be the HCPB blanked developed at KIT in the frame of the Power Plant Physics and Technology Programme (PPPT) under EFDA, but that takes into account a recent update conducted in 2014 with the EUROfusion PPPT programme [9]. The blanket design for all three blanket concepts is the same.

The DEMO-tokamak is divided into $22.5^{\circ}$ symmetric sectors $\left(16\right.$ in total $\left.{ }^{1}\right)$. For the in-vessel components (First Wall, Blanket and Manifold) each sector is composed by 2 inboard and 3 outboard segments [3]. The outboard segments consist of a Central Outboard Blanket Segment (COBS) between the toroidal field coils, a Right and a Left Outboard Blanket Segments (ROBS and LOBS respectively) each partly under a toroidal field coil. Both outboard and inboard segments contain 6 modules. Figure 1 shows the corresponding CAD model into which the ICRF antenna has been integrated. Because of symmetries, only $11.25^{\circ}$ are considered to simplify the simulations.

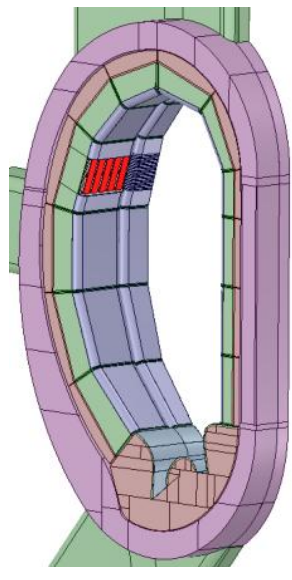

FIGURE 1. DEMO torus sector model with blanket modules and distributed antenna integrated (Faraday screen partially removed)

The model includes the in-vessel components (breeding blanket modules with manifolds and shields, the vacuum vessel, the divertor and the ports). The contribution of the toroidal and poloidal coils are neglectable when calculating the TBR. The First Wall of the blanket modules consist of $2 \mathrm{~mm}$ tungsten on top of $25 \mathrm{~mm}$ cooled Eurofer. The breeding material consists of a homogeneous composition of 57\% Beryllium (neutron multiplier) + $19 \% \mathrm{Li}_{4} \mathrm{SiO}_{4}$ (ceramic breeder with ${ }^{6} \mathrm{Li}$ enriched up to at. 60\%) $+15 \%$ EUROFER (cooling plates and stiffening grid) $+9 \%$ He (coolant). Behind the breeding zone a cooled Eurofer mixture is used for both the backplates of the blanket and the manifold.

\footnotetext{
${ }^{1}$ This was the design for which the calculation was made. The present design considers 18 coils but this will not affect the fundamental result.
} 


\section{DISTRIBUTED ANTENNA}

To be acceptable the antenna must comply with the following conditions [10]:

- The antenna must not impair any blanket function: it has to keep ensuring the tritium self-sufficiency and the capability to extract the energy from the fusion reaction. The effect in other blanket functions is not studied in this work.

- The antenna must match the blanket modularity and not require extra openings in the vessel: each blanket module is independent from the others, so the antenna can't require an internal connection between modules and it must use one of the already existing ports for the feeding lines.

- The antenna must share the same coolant of the blanket: He or water, the cooling system must be integrated with the one of the blanket.

- The antenna must not increase the complexity of the Remote Handling: the remote handling of the blanket is a complex system through the upper port where the pipes and tubes are welded and rewelded outside the VV [11]. Hence, the remote handling for the antenna must work in the same way.

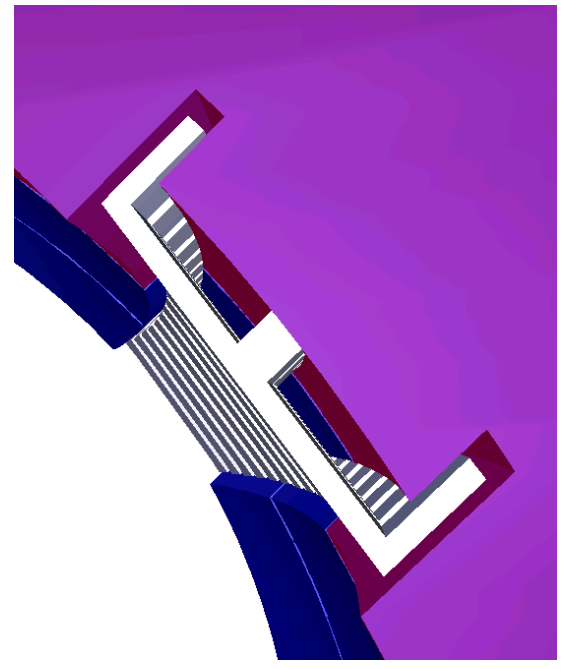

Figure 2. Draft of the reference ICRF antenna integrated in a DEMO reactor
The detailed configuration of the antenna is still being defined, and in particular the feeding of the antenna. A reference antenna was made with the following assumptions [12]:

The reference antenna is a toroidal $360^{\circ}$ ring array of straps embedded in a slot as can be seen in Figure 2.

The slot is C-shaped and $100 \mathrm{~cm}$ high. The radial thickness is $15 \mathrm{~cm}$ except for the capacitor holes located in the upper and lower part, which are $20 \mathrm{~cm}$ thick (radially).

Within the slot, the straps of the ring array are $96 \mathrm{~cm}$ high (poloidally), $20 \mathrm{~cm}$ wide (toroidally), and $2 \mathrm{~cm}$ deep (radially). Between straps there is a separation of $6 \mathrm{~cm}$, thus the area of the straps over the total area of the antenna, defined as covering ratio in Chapter 4 , is $72 \%$. As can be seen in Figure 2, not all the space of the antenna is filled with the straps. In each of the 3 blanket segments composing the blanket sector (a sector is $22.5^{\circ}$ of the whole tokamak) the antenna has 5 straps, thus there are 15 straps per blanket sector (Figure 3).

In front of the straps there is the Faraday Screen (FS) flush with the $\mathrm{FW}$, as can be seen in Figure 3. The tentative poloidal dimensions are

For the material of the antenna, the same material is used as for stiffening the blanket (Eurofer). Alternatively, due to its position near the plasma, tungsten may be required, although the presence of a Faraday screen may alleviate this. For the time being the feeding lines through the blanket have been neglected. For the Faraday screen the same material as the First Wall has been used ( $2 \mathrm{~mm}$ of tungsten and $25 \mathrm{~mm}$ of He-cooled Eurofer). Hence, the neutrons have to face first the FS (2,7 cm thick) and then the antenna ( $2 \mathrm{~cm}$ thick).

In every blanket segment the antenna is using only one module, which is in the same poloidal position than in the rest of the segments. The total First Wall area used for the antenna over the whole tokamak is $67 \mathrm{~m}^{2}$.

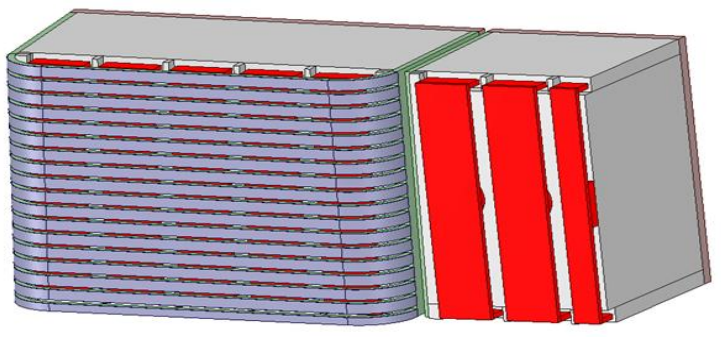

(a)

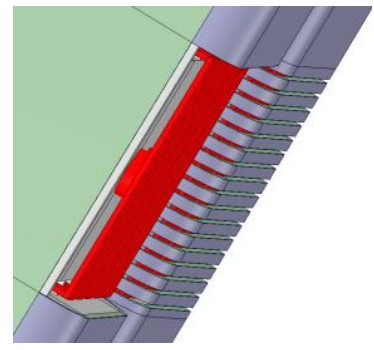

(b)

FIGURE 3. Antenna (Faraday Screen partially removed): separated from the rest of the blanket (a) and integrated in the blanket (b). It corresponds to 1,5 blanket segments 


\section{CAlCulations}

As said above, the DEMO model developed at KIT for the HCPB blanket was used as the reference model. The CAD model of the ICRF antenna was integrated into this model using the SpaceClaim software [13]. The resulting CAD geometry model was then converted into MCNP geometry using KIT's McCad interface [14]. The calculations were carried out using the MCNP-5 code and the FENDL-2.1 nuclear data library on $11.25^{\circ}$ torus sector while taking symmetries into account. The track length estimator F4 (track length estimator) [6] was used to calculate the TBR. Typically 100 million neutron source histories were tracked in a MCNP run to get sufficient statistics. The results for the reference configuration defined previously are shown first and a parametric analysis on different parameters is done later.

\subsection{Reference configuration}

Table 1 shows the neutron balance and the effect on the TBR of the considered cases. When two numbers appear in the table, the left one refers to the mean and the right one to the error in percentage from the mean. Although the antenna takes up $360^{\circ}$ around the torus, the breeding volume removed is small, and so it is the loss of TBR.

TABLE 1. Results of the MCNP-5 calculations for a DEMO with HCPB blanket

\begin{tabular}{cccc}
\hline & Without antenna & With antenna & Variation (\%) \\
\hline Breeding volume per blanket segment $\left(\mathrm{m}^{3}\right)$ & 23.89 & 23.64 & $-1.055 \%$ \\
Net multiplication & 1.6130 .0001 & 1.6090 .0001 & $-0.248 \%$ \\
Total capture (per source neutron) & 1.609 & 1.605 & $-0.249 \%$ \\
Capture in breeding material (per source & 1.3210 .0003 & 1.3160 .0003 & $-0.379 \%$ \\
neutron) & 1.1450 .0003 & 1.1410 .0003 & $-0.349 \%$ \\
Tritium Breeding Ratio & & &
\end{tabular}

As one can see from Table 1, the effect of the antenna on the neutron multiplication and the TBR is small. The reason are that, the parasitic absorption in the antenna is small, the change of neutron spectrum is rather a positive effect, and the breeder volume is not reduced by much. With the assumed HCPB breeder blanket, the relative reduction of the TBR is lower than the reduced breeding volume (though the antenna sits in front, for the reduction of volume it is as if a volume in the back, the least productive, is removed). Such low impact on the TBR, together with the fact that the antenna does not need to be placed in a port, enhances the attractiveness of the ICRF and makes possible to combine ICRF with other heating methods or diagnostics that do use the equatorial ports. An interesting comparison for these results is with an equivalent void opening in the equatorial port, which is what is typically used by other auxiliary heating systems. Taking 16 ports with $1 \mathrm{~m} \times 2 \mathrm{~m}$ openings ( $32 \mathrm{~m}^{2}$ in total) the loss of TBR in an HCBP blanket is $10 \%$ [2]. The effect of the complete ICRF antenna (total area of $\sim 67 \mathrm{~m}^{2}$ ) is thus equivalent to a single void opening of $1.40 \mathrm{~m}^{2}$ in the equatorial port.

\subsection{Parametric analysis}

DEMO is still in a preliminary phase and therefore it is still under development. ITER will be the key milestone with the opportunity to test and improve many features, but that also means that there is still a wide range of possibilities concerning different aspects. The results so far have shown that the antenna concept is feasible in terms of tritium self-sufficiency in the current configuration. Since the design is in a preliminary stage, it is important to do a parametric analysis to see how the loss of TBR changes due to the antenna if some of the parameters are modified.

The parameters studied here are the ones which meet the criteria of both an apparently strong dependence with the TBR on one hand, and the ones which are more likely to change in a near future on the other hand. The objective, then, is to provide guidance as to which factor affect mostly the TBR and thus also provide guidance for further antenna design and allow to estimate the loss of TBR in a short and middle term horizon. 
Blanket concept. The HCPB blanket composition in the blanket modules has been replaced first by a HCLL $(85 \% \mathrm{~Pb}-\mathrm{Li}+8 \%$ Eurofer $+7 \%$ void $)$ and then by a WCLL $(80 \% \mathrm{~Pb}-\mathrm{Li}+18 \%$ Eurofer $+2 \%$ Water $)$ type mixture to study the antenna effect on the TBR for blankets with different neutronics characteristics. Table 2 shows the neutron balance for these cases. It is revealed that the strongest effect regarding the blanket concept is for the HCLL mixture, with $-0.53 \%$ (TBR variation), but it is still low. The WCLL is in the middle of the three blanket concepts with a loss TBR of $-0.51 \%$ but closer to the HCLL. These results were actually expected since the use of the HCLL mixture results in a faster neutron spectrum and thus, as opposed to the HCPB mixture, results in an enhanced neutron out-scattering losses from the breeder and increased parasitic absorptions in the structure including the antenna. The WCLL is lower than the HCLL due to the higher moderation and absorption of water rather than helium.

TABLE 2. Variation between the reactor with and without the antenna implemented for the HCPB, HCLL and WCLL blanket concepts

\begin{tabular}{cccc}
\hline & HCPB & HCLL & WCLL \\
\hline Breeding volume per blanket segment $\left(\mathrm{m}^{3}\right)$ & $-1.055 \%$ & $-1.055 \%$ & $-1.055 \%$ \\
Tritium Breeding Ratio & $-0.349 \%$ & $-0.532 \%$ & $-0.507 \%$ \\
\hline
\end{tabular}

Covering Ratio. The covering ratio of the straps is defined as the ratio between the surface of all the straps and the projected surface of the antenna slot. The covering ratio of the antenna studied is $\sim 72 \%$. Due to the U-shape

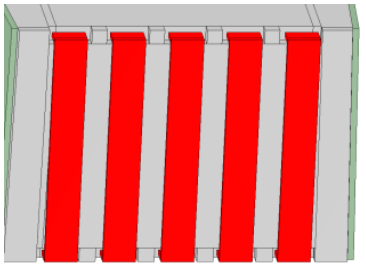

(a)

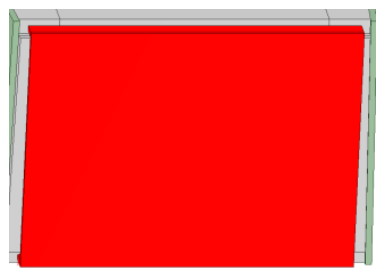

(b)

FIGURE 4. Covering ratio of the antenna straps: $49 \%$ (a) and $94 \%$ (b) of the $\mathrm{FW}$, the maximum covering ratio with the current antenna-shape is $\sim 94 \%$ (see Fig. 4-b). A third calculation with a $\sim 49 \%$ covering ratio is done to complement the sensitivity analysis (see Fig. 4-a). Results are shown in Table 3. These results reveal that the covering ratio strongly affects the loss of TBR, but even in the worst case ( $94 \%$ covering ratio) the loss remains low with $-0.44 \%$.

TABLE 3. Results for sensitivity analysis on the covering ratio of the antenna (DEMO HCPB)

\begin{tabular}{|c|c|c|c|c|c|c|c|}
\hline & \multirow{2}{*}{$\begin{array}{c}- \\
\begin{array}{l}\text { Without } \\
\text { antenna }\end{array}\end{array}$} & \multicolumn{2}{|c|}{ 49\% Covering Ratio } & \multicolumn{2}{|c|}{$72 \%$ Covering Ratio } & \multicolumn{2}{|c|}{ 94\% Covering Ratio } \\
\hline & & $\begin{array}{c}\text { With } \\
\text { antenna }\end{array}$ & Variation & $\begin{array}{c}\text { With } \\
\text { antenna }\end{array}$ & Variation & $\begin{array}{c}\text { With } \\
\text { antenna }\end{array}$ & Variation \\
\hline $\begin{array}{l}\text { Breeding volume per } \\
\text { blanket segment }\left(\mathrm{m}^{3}\right)\end{array}$ & 23.890 & 23.641 & $-1.042 \%$ & 23.638 & $-1.055 \%$ & 23.637 & $-1.059 \%$ \\
\hline Tritium Breeding Ratio & 1.145 & 1.143 & $-0.175 \%$ & 1.141 & $-0.349 \%$ & 1.140 & $-0.437 \%$ \\
\hline
\end{tabular}

Antenna radial thickness. The radial thickness of the antenna is the total thickness of the breeding blanket that is occupied by the antenna. Not all this thickness is removed from the breeding blanket because there are the capacitor holes in the upper and lower part of the strap which are $5 \mathrm{~cm}$ deeper than the rest. The reference configuration of the antenna is $20 \mathrm{~cm}$ thick (radial direction) and that reference calculation is complemented with another one for a $40 \mathrm{~cm}$ thick case. The shape and size of the antenna is not modified, only the volume of blanket removed. It is a very conservative case, because the radial thickness of the antenna is not very likely to be changed by that much. This very conservative case though helps to understand the effect of the radial thickness and acts, at the same time, as the worst case. In Table 4 the results for both thicknesses are shown. 
Table 4. Results for sensitivity analysis on the radial thickness of the antenna (DEMO HCPB)

\begin{tabular}{ccc}
\hline & $\mathbf{2 0} \mathbf{~ c m}$ & $\mathbf{4 0} \mathbf{~ c m}$ \\
\hline Breeding volume per blanket segment $\left(\mathrm{m}^{3}\right)$ & $-1.055 \%$ & $-2.817 \%$ \\
Tritium Breeding Ratio & $-0.349 \%$ & $-0.524 \%$ \\
\hline
\end{tabular}

The results show that when doubling the radial thickness of the antenna up to $40 \mathrm{~cm}$ the loss of TBR remains low with $-0.52 \%$. The effect is less than linear.

Straps Thickness. This parameter is directly related with the parasitic absorption of neutrons because it varies the amount of material between the plasma and the breeding blanket that do not contribute to the TBR. The reference configuration of the antenna uses straps $2 \mathrm{~cm}$ thick and this case is complemented with two more. The first one, following the same idea as in the case of the antenna depth, using a very conservative case, a $4 \mathrm{~cm}$ thick straps is implemented. The second one uses a strap with the composition of the FW. That is, $2.5 \mathrm{~cm}$ Eurofer plus $0.2 \mathrm{~cm}$ Tungsten. Results are shown in Table 5. This second case would be interesting if the antenna required more shielding that the one provided by the Faraday Screen.

Table 5. Results for sensitivity analysis on the thickness of the straps (DEMO HCPB)

\begin{tabular}{cccc}
\hline & $\mathbf{2 ~ c m}$ & $\mathbf{2 . 5}+\mathbf{0 . 2} \mathbf{~ c m}$ & $\mathbf{4} \mathbf{~ c m}$ \\
\hline Breeding volume per blanket segment $\left(\mathrm{m}^{3}\right)$ & $-1.055 \%$ & $-1.063 \%$ & $-1.067 \%$ \\
Tritium Breeding Ratio & $-0.349 \%$ & $-0.437 \%$ & $-0.611 \%$ \\
\hline
\end{tabular}

The results show that when doubling the thickness of the straps the loss of TBR increases up to $-0.61 \%$. It is the worst case of all the parametric analysis but it is still a low value and still guarantees the TBR to stay above the unity. The case with $2.5+0.2 \mathrm{~cm}$ with the composition of the FW shows a loss of TBR of $-0.44 \%$.

Poloidal Position. The last parameter studied is the poloidal position of the antenna in the radial-poloidal cross section of the torus (see Figure 5). The final position will be defined by the functionality of the antenna and it is still being studied. Anyway, it is already set that it will be placed in the outboard upper modules, so modules 3, 4, 5 and 6 are the candidates. The reference configuration has the antenna placed in module 4 .

The effect on the loss of TBR of this parameter is directly related to the Neutron Wall Loading (NWL) because the NWL is an indicator of the amount of neutrons in the region on which the antenna will have an impact. The larger the NWL, the larger the loss of TBR. Hence, module 3 is the worst case in terms of TBR,

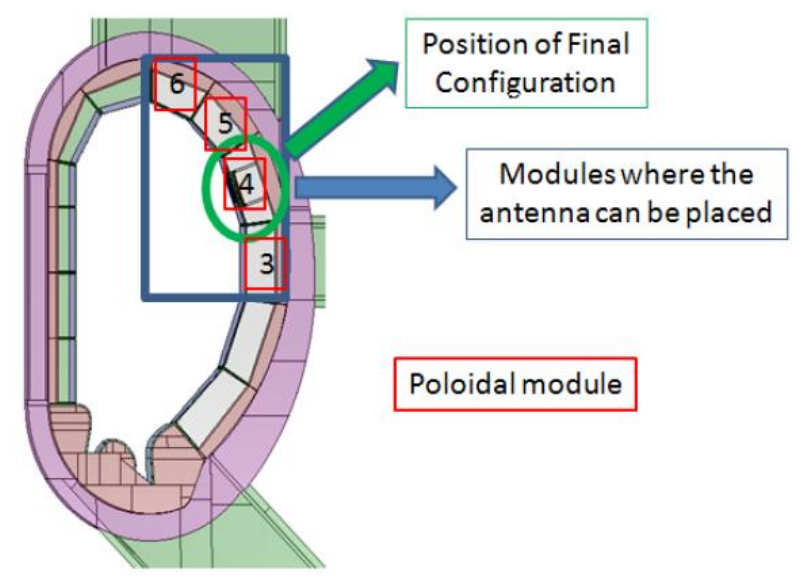

FIGURE 5. Poloidal availability for the reference antenna which corresponds to $0^{\circ}$ (or $360^{\circ}$ ), and, on the outside, module 6 is the best, which corresponds to $90^{\circ}$. Both cases have been studied to complement the reference configuration, which places the antenna in module 4 , corresponding to $40^{\circ}$. Results are shown in Table 6 . 
Table 6. Results for sensitivity analysis on the Poloidal Position (DEMO HCPB)

\begin{tabular}{cccc}
\hline & Module 3 & Module 4 & Module 6 \\
\hline Breeding volume per blanket segment $\left(\mathrm{m}^{3}\right)$ & $-1.088 \%$ & $-1.055 \%$ & $-0.850 \%$ \\
Tritium Breeding Ratio & $-0.349 \%$ & $-0.349 \%$ & $-0.175 \%$ \\
\hline
\end{tabular}

The breeding volume reduction is not the same in all the modules because of geometric reasons. For smaller blanket modules the implementation of the antenna uses less volume because it needs to be adapted to the corresponding module.

Of the available modules for placing the antenna, module 6 is indeed the best case with a loss on the TBR of $-0.18 \%$. The NWL there is the lowest with a value of $0.9 \mathrm{MW} / \mathrm{m}^{2}$ (see Figure 6 ).

The worst case, with a NWL of $1.35 \mathrm{MW} / \mathrm{m}^{2}$ (see Figure 6 ), is module 3 with a loss on the TBR of $-0.35 \%$. The interesting part is that the loss on the TBR of modules 3 and 4 is not significantly different.

The explanation for these results is that no big difference in NWL is observed for module 4 and 3 , which are $1.2 \mathrm{MW} / \mathrm{m}^{2}$ and $1.35 \mathrm{MW} / \mathrm{m}^{2}$ respectively.

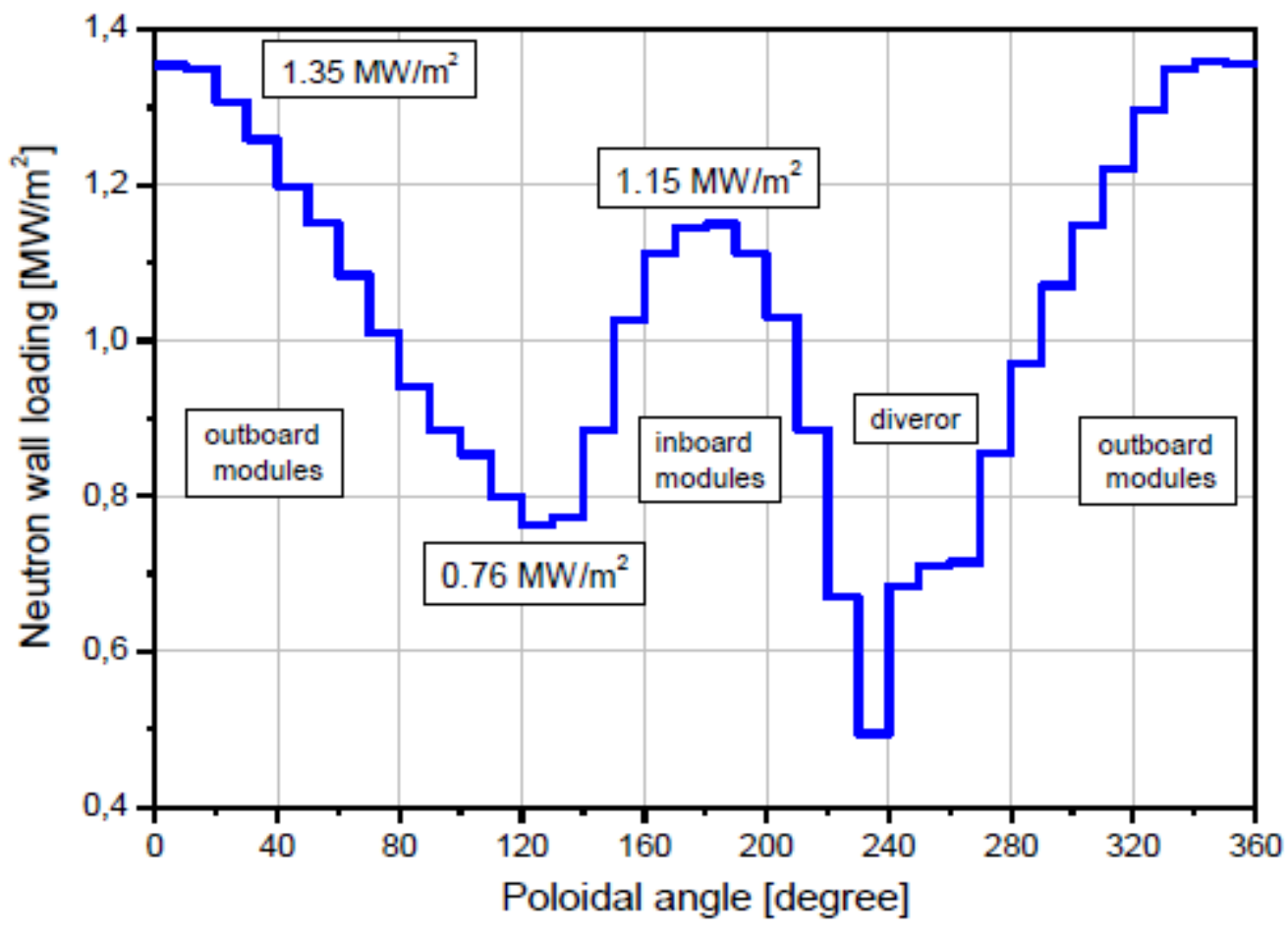

Figure 6. Neutron Wall Loading (NWL) in HCPB DEMO reactor [15] 


\section{CONCLUSIONS}

A quantification of the loss of TBR for a distributed antenna in a DEMO reactor has been performed in this paper based on Monte Carlo calculations. The ICRF distributed antenna was shown to have only a small effect on the tritium breeding performance of DEMO. For a DEMO with HCPB blanket, there is only a reduction $0.35 \%$ of the TBR, which is equivalent to a void port opening on the equatorial plane of $1.4 \mathrm{~m}^{2}$. The reduction is bigger for the HCLL blanket concept $(0.53 \%)$ than for the HCPB $(0.35 \%)$. The TBR reduction strongly depends on the covering ratio of the antenna, but is always below $0.44 \%$. The reduction of the TBR when doubling the radial thickness of the antenna up to $40 \mathrm{~cm}$ is $-0.52 \%$. The thickness of the straps has largest impact on the TBR and increases the loss up to $-0.61 \%$ with a thickness of $4 \mathrm{~cm}$ and up to $-0.44 \%$ when implementing a mixed composition of $2.5 \mathrm{~cm}$ He-cooled Eurofer $+0.2 \mathrm{~cm} \mathrm{~W}$, the composition of the FW. Finally, the TBR goes from $-0.35 \%$ in the equatorial port to $-0.18 \%$ in the upper port, the best and worst cases respectively for the poloidal position of the antenna.

The low results show that the tritium self-sufficiency will not be a fundamental problem in the development of a distributed - in blanket ICRF antenna for DEMO.

\section{ACKNOWLEDGMENTS}

This work has been carried out within the framework of the EUROfusion Consortium and has received funding from the Euratom research and training programme 2014-2018 under grant agreement No 633053. The views and opinions expressed herein do not necessarily reflect those of the European Commission.

\section{REFERENCES}

1. G. Federici, R. Kemp, D. Ward. C. Bachmann, T. Franke et al, Overview of EU DEMO design activities, Fus. Eng. Des. 89(2014), 882-889

2. U. Fischer, Neutronics Requirements for a DEMO Fusion Power Plant, Fusion Eng. Design (2015)DOI: 10.1016/j.fusengdes.2015.02.029

3. G. Bosia, "Low power density Ion Cyclotron Arrays for Fusion Reactors" Fusion Engineering and Design, 92(2015), 8-15

4. L.V. Boccaccini, Design And Development of Demo Blanket Concepts in Europe, 1st IAEA DEMO Programme Workshop, UCLA, $15-18$ Oct. 2012

5. U. Fischer, Neutronics R\&D Efforts in Support of the European Breeder Blanket Development ProgrammeDOI: 10.1088/0029-5515/49/6/065009

6. X-5 Monte Carlo Team, MCNP-A General Monte Carlo N-Particle Transport Code (Version 5, Vol. I), Report LA-UR-031987, 24 April 2003 (Revised 10/3/05).

7. D.L. Aldama, A. Trkov, FENDL-2.1: update of an evaluated nuclear data library for fusion applications, Report INDC(NDS)-467, December 2004.

8. L.V. Boccaccini, et al., Materials and design of the European DEMO blankets, Journal of Nuclear Materials 329-333 (2004) 148-155

9. P. Pereslavtsev, et al., Neutronic analyses of the HCPB DEMO reactor using a consistent integral approach, Fusion Eng. Des. (2014), http://dx.doi.org/10.1016/j.fusengdes.2013.12.019

10. G. Bosia, A Ring Array for $H \& C D$ in DEMO, H\&CD Intermediate Meeting on IC (4 Sept 2014)

11. D. Iglesias, et al., Blanket Segment Remote Maintenance, Report for Task Agreement WP13/DAS07/T05

12. Ricardo Ragona, ERM/KMS Brussels, personal communication (2015)

13. 3D Modeling Software for Engineering SpaceClaim, http://www.spaceclaim.com

14. L. Lu, U. Fischer, P. Pereslavtsev, Improved Algorithm and Advanced Features for the CAD to MC Conversion Tool McCad, Fusion Eng. and Design 89(2014), 1885-1888.

15. PERESLAVTSEV, P. Nuclear analysis of breeder blanket for HCPB DEMO. Neutronics coordination meeting. December 2014b. 